\title{
Tank Based Unified Torque/Impedance Control for a Pneumatically Actuated Antagonistic Robot Joint
}

\author{
Alexander Toedtheide, Erfan Shahriari and Sami Haddadin ${ }^{1}$
}

\begin{abstract}
In this paper the concept of a unified torque/impedance controller is applied to a pneumatically actuated, antagonistic robot joint. The investigated control algorithm consists of a cascaded structure in which the outer torque/impedance controller commands a desired torque to two cylinder-based force controllers. The torque-/impedance controller is equipped with a virtual tank that ensures passivity of the control loops above force level. Additionally, a shaping function provides a continuous transition to impedance control in case of sudden contact loss during a torque control operation. External torques used in the feedback loop for contact force regulation are estimated by a momentum observer. Experimental and simulation results show a maximum deflection of $9.7^{\circ}$ over $130 \mathrm{~ms}$ in case of contact loss until a resting position is reached. Additionally, step and sinusoidal torque tracking up to $5 \mathrm{~Hz}$ was successfully tested at good performance.
\end{abstract}

Index Terms-compliance and impedance control, hydraulic/pneumatic actuators, tendon/wire mechanism

\section{INTRODUCTION}

In order for a robot to interact with and manipulate in the real world compliant robot mechanisms and force sensitive controls are required.

Compliant elements, when considering elastic joints and no structural compliances, may be e.g. series elastic actuators (SEA) [1] or variable stiffness actuators (VSA) [2] with passive spring systems in the joint. More recently also various pneumatic systems were developed [3], [4], [5]. They are inherently compliant, lightweight and have a high powerto-weight-ratio. Furthermore, they can store energy, show robust impact absorption and require no gearing.

Nowadays, the most commonly used scheme for forcesensitive control is impedance control, dating back to the original work of Hogan [6]. In its most common form it lets the robot behave like a mass-spring-damper system with respect to interaction forces. Therefore, maximum contact forces may be limited within the control bandwidth by selecting suitable impedance settings.

Apart from compliant control, the accurate regulation of contact forces is typically of large interest during grasping and manipulation. Force control is generally divided into direct and indirect force control [7]. When using impedance control to apply desired forces to objects and the environment, according set-point motions are used that make use of the closed loop impedance relation. Since impedance control is not designed to accurately control contact forces it is an indirect method. Precise force tracking can only be achieved by accurately knowing contact geometry and

This work has been partially funded by the European Union's Horizon 2020 research and innovation programme under grant agreement No 688857, and by the Alfried-Krupp Award for young professors. ${ }^{1}$ All authors are with Institute of Automatic Control. Faculty of Electrical Engineering and Computer Science, Institute of Automatic Control, Leibniz Universität Hannover, Appelstr. 11 D-30167 Hannover, Germany \{toedtheide, shahriari, sami.haddadin\}eirt.uni-hannover.de contact mechanics. A good summary with according stability analysis on existing direct force control methods, which make use of measured or estimated [16] contact forces, can be found in [8]. For pneumatic actuators force, stiffness [3], [9], [4], [5] and impedance control [10], [11], [5], [13] were also intensively investigated over the last years.

Problem: During the execution of contact tasks both, force and impedance control, may become potentially dangerous in case of a sudden contact loss: For force control the constant force error lets the system drive towards the desired force direction at increasing speed. For impedance control, the potential energy stored in the virtual springs during execution of the contact task is released instantaneously and transformed into potentially dangerous, however, at least unwanted kinetic energy.

Contribution: The control scheme from [12], which was designed for torque controlled robots, is the first provably stable unification of force and impedance control, while also safely handling the contact-loss problem. In this paper, we apply the methods to antagonistically driven pneumatic robot joints [13] that provide backdriveability and joint torque measurement based on pressure sensing. To the best of the authors' knowledge no previous publications dealt with the investigation of unified torque-/force impedance control and safe contact-loss handling in the domain of pneumatic actuators.

The main contributions of the present paper are

- the application of the momentum based disturbance observer paradigm to pneumatic systems for estimating external torques without acceleration measurements,

- basic feedback control of estimated contact torques for an antagonistic pneumatic actuator,

- application of unified torque and impedance control framework to pneumatically actuated robot joints and

- providing a first step towards a full passivity analysis of the closed loop pneumatic system.

The remainder of this paper is organized as follows. Section II describes the modeling of the pneumatic actuator. In Section III the control algorithms including generalized momentum observer are summarized and an initial passivity analysis is performed. Section IV presents simulative and experimental results. Finally, the paper concludes in Section V.

\section{System Modeling}

Figure 1 shows the scheme of the antagonistic and pneumatically actuated robot joint. It consists of two double chambered pneumatic cylinders, each one equipped with linearly moving pistons, whose positions are denoted by $x_{\mathrm{p} 1}$ and $x_{\mathrm{p} 2}$, respectively. Each piston is connected to a tendon, which transmits the force $F_{\mathrm{t} i}$ to a roller pulley. The tendons are assumed rigid, since the maximum theoretical elongation 
amounts to $0.12 \mathrm{~mm}$ [13]. The tendons are attached at radius $r$. The relation between roller pulley angle $q$ and piston position $x_{\mathrm{p} i}$ is simply $q r=-x_{\mathrm{p} 1}=x_{\mathrm{p} 2}$. It is assumed that during operation no tendon slacking occurs. See a more detailed higher order modeling with a flexible joint description in our previous work [13].

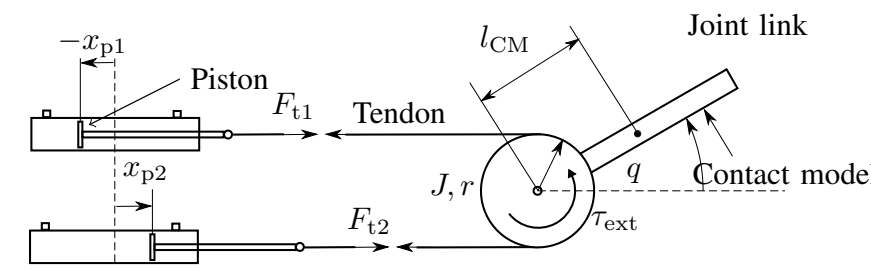

Pneumatic cylinder

Fig. 1: Mechanical model of the antagonistic pneumatic joint

The motion of the system is described by

$$
J_{\mathrm{c}} \ddot{q}+M_{\mathrm{j}} l_{\mathrm{CM}} \cos (q)=r\left(F_{\mathrm{t} 1}-F_{\mathrm{t} 2}\right)+\tau_{\mathrm{ext}},
$$

where $J_{\mathrm{c}}=\left(J+2 M_{\mathrm{P}} r^{2}\right)$ is the effective inertia of roller pulley $J$ and piston masses $M_{\mathrm{p}}$. Gravity influences are characterized by the the mass of the roller pulley $M_{\mathrm{j}}$ and the distance to the center of mass $l_{\mathrm{CM}}$. External torques are denoted as $\tau_{\text {ext }}$. Friction is neglected. Each tendon force

$$
F_{\mathrm{t} i}=-P_{i 1} A_{1}+P_{i 2} A_{2}+P_{\mathrm{atm}} A_{\mathrm{r}}
$$

is affected by the pressure $P_{i 1}$ and $P_{i 2}$ of the right and left chamber of the pneumatic cylinder multiplied by the effective cylinder areas $A_{1}$ and $A_{2}$. Due to the rod, leading out of chamber 2, the product of ambiance pressure $P_{\mathrm{atm}}$ and cross section $A_{\mathrm{r}}$ induce a force to the piston, see Fig. 2. Consequently, the relation $A_{2}=A_{1}-A_{\mathrm{r}}$ holds.

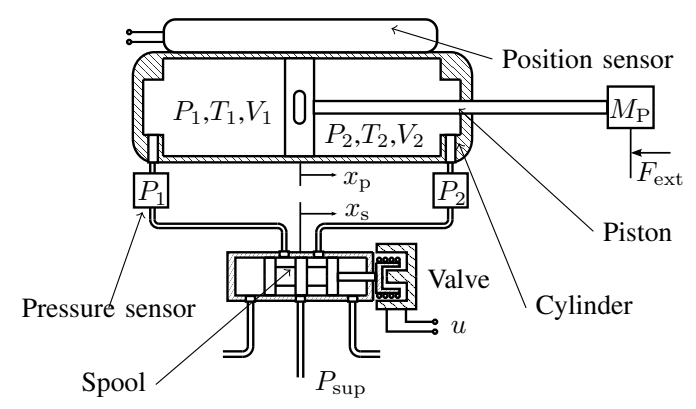

Fig. 2: Technical drawing of the pneumatic system [13]

The pressure dynamics of a cylinder chamber can be described by its pressure dynamics ${ }^{1}$

$$
\dot{P}=\frac{R T_{\text {atm }}}{V}\left(\alpha_{\text {in }} \dot{m}_{\text {in }}-\alpha_{\text {out }} \dot{m}_{\text {out }}\right)-\alpha \frac{P}{V} \dot{V}
$$

which was described in [14]. The parameter $R$ is the specific gas constant of air and $T_{\text {atm }}$ is the ambient temperature. $\alpha$, $\alpha_{\text {in }}$ and $\alpha_{\text {out }}$ are parameters which are used to improve the model fitting for piston motion, inflow and outflow due to

${ }^{1}$ For better readability the indices for multiple cylinders are omitted. the assumption of constant temperature $T_{\mathrm{atm}}$. The volume of a chamber is $V=V_{0}+\left(L / 2 \pm A_{i} x_{\mathrm{p}}\right)$, where $L$ is the stroke of the cylinder and $V_{0}$ is the dead volume. The valve is modeled by

$$
\dot{m}=c_{\mathrm{f}} A\left(x_{\mathrm{s}}\right) P_{\mathrm{u}} \Psi\left(P_{\mathrm{d}} / P_{\mathrm{u}}\right),
$$

where $P_{\mathrm{u}}$ and $P_{\mathrm{d}}$ are up- and downstreams with $P_{\mathrm{u}}>$ $P_{\mathrm{d}}$. Additional case distinctions are required to allocate $P_{\mathrm{u}}$ and $P_{\mathrm{d}}$ to $P_{i}, P_{\mathrm{atm}}$ and $P_{\mathrm{sup}}$ for in- and outflow. $c_{\mathrm{f}}$ is an attenuation factor and $\Psi\left(P_{\mathrm{d}} / P_{\mathrm{u}}\right)$ is a dimensionless flow function [13], [14]. $A\left(x_{\mathrm{s}}\right)$ describes the orifice area in dependency of the valve spool position $x_{\mathrm{s}}$ which can be identified experimentally. A linear relation $x_{\mathrm{s}}=K u$ between spool position $x_{\mathrm{s}}$ and valve input voltage $u$ is assumed.

\section{CONTROL}

In this section, the impedance control, torque control and passivity based unified torque-/impedance control are described. These controllers are part of a cascaded control structure which inner loop consists of a sliding mode force controller for each pneumatic cylinder based on [17] which controls the force applied to the piston of each cylinder. The detailed operation of this controller can be found in our previous work [13]. For this reason we consider the pneumatic cylinders as force sources which ideally control the tendon forces $F_{\mathrm{t} 1}$ and $F_{\mathrm{t} 2}$ to track the desired forces $F_{\mathrm{d} 1}$ and $F_{\mathrm{d} 2}$.

\section{A. Antagonistic system}

As the inner force control loop operates on force level of the cylinder pistons, while the following controllers act on joint level, a transformation between rotational and linear units is required. The tendon Jacobian $\boldsymbol{P}(q)$ describes the relation between joint velocities and linear motion as well as tendon force and joint torques [18], [19]:

$$
\dot{\boldsymbol{x}}_{\mathrm{p}}=\boldsymbol{P}(q)^{T} \dot{q}, \tau=\boldsymbol{P}(q) \boldsymbol{F}_{\mathrm{t}}
$$

with $\boldsymbol{F}_{\mathrm{t}}=\left(F_{\mathrm{t} 1}, F_{\mathrm{t} 2}\right)^{T}$. From [13] the tendon Jacobian is known to be

$$
\boldsymbol{P}(q)=\left(\begin{array}{ll}
-1 & 1
\end{array}\right) r
$$

Given a desired torque $\tau_{\mathrm{d}}$ the desired forces on piston level $\boldsymbol{F}_{\mathrm{d}}=\left(F_{\mathrm{d} 1}, F_{\mathrm{d} 2}\right)^{T}$ can be obtained from

$$
\boldsymbol{F}_{\mathrm{d}}=\boldsymbol{P}^{\dagger} \tau_{\mathrm{d}}+\boldsymbol{F}_{\mathrm{P}},
$$

where $\boldsymbol{P}^{\dagger}$ denotes the right pseudo inverse of $\boldsymbol{P}(q)$ and $\boldsymbol{F}_{\mathrm{P}}$ is a vector of constant pretension forces. Given the vector of piston positions $x_{\mathrm{P}}=\left(x_{\mathrm{P} 1}, x_{\mathrm{P} 2}\right)^{T}$ the joint angle $q$ is estimated by ${ }^{2}$

$$
q=\boldsymbol{P}^{\dagger^{T}} \boldsymbol{x}_{\mathrm{P}}
$$

\section{B. Joint level impedance control}

In [13] we used the impedance controller

$$
\tau_{\mathrm{d}, \mathrm{imp}}=k_{\mathrm{imp}}\left(q_{\mathrm{d}}-q\right)-d_{\mathrm{imp}} \dot{q}+\hat{g}(q)
$$

of the antagonistic system. Here, $\tau_{\mathrm{d}, \text { imp }}$ is the desired torque of the controller applied on joint level, $q_{\mathrm{d}}$ is the desired angle, and $k_{\mathrm{imp}}$ and $d_{\mathrm{imp}}$ are stiffness and damping. Additionally, $\hat{g}(q)$ is the compensation of gravity.

\footnotetext{
${ }^{2}$ In principle one position sensor can be used to calculated the angle $q$. However, the use of two sensors and (8) enables an improved angular estimation.
} 


\section{Joint level torque control}

Joint torque control for the antagonistic pneumatic system is achieved by a PID controller

$$
\begin{aligned}
\tau_{\mathrm{d}, \mathrm{trq}} & =\alpha\left(K_{\mathrm{p}} e+K_{\mathrm{d}} \dot{e}+\int_{t_{1}}^{t} K_{\mathrm{i}} e d \sigma\right) \\
e & =\tau_{\mathrm{d}, \mathrm{f}}-\hat{\tau}_{\mathrm{ext}}
\end{aligned}
$$

with the controller parameters $K_{\mathrm{p}}, K_{\mathrm{i}}$ and $K_{\mathrm{d}}$ for the proportional, integral and derivative part. $\tau_{\mathrm{d}, \mathrm{f}}$ is the desired torque at joint level and $\hat{\tau}_{\text {ext }}$ denotes an observed external torque (see Sec. III-D) which is used for the torque error $e$. The parameter $\alpha=[0,1]$ enables an on/off switching of the controller. $t_{1}$ resets the integral part.

\section{Generalized momentum observer}

The external torque $\tau_{\text {ext }}$, required for our torque control (11), is estimated by an observer for the generalized momentum [15], [16] with

$$
\hat{\tau}_{\mathrm{ext}}=K_{\mathrm{O}}\left(\int_{0}^{T}\left(\tau_{\mathrm{m}}-M_{\mathrm{j}} l_{\mathrm{CM}} \cos (q)-\hat{\tau}_{\mathrm{ext}}\right) d t-J_{\mathrm{c}} \dot{q}\right)
$$

with the observer gain $K_{\mathrm{O}}$. The motor torque is estimated by $\tau_{\mathrm{m}}=r\left(F_{\mathrm{t} 1}-F_{\mathrm{t} 2}\right)$ and (2) using the pressure sensors of the four cylinder chambers.

\section{E. Passivity based unified torque/impedance controller}

Based on (9) and (10) a preliminary unified torque/impedance control for the pneumatic joint can be built as

$$
\tau_{\mathrm{d}}^{\prime}=\tau_{\mathrm{d}, \operatorname{trq}}+\tau_{\mathrm{d}, \mathrm{imp}}
$$

For the stability analysis of the controller, a passivity-based approach is used, which is defined as follows:

Theorem: (Passivity)

Considering the state space model of a standard nonlinear system $\dot{x}=f_{s s}(x, u)$ with output $y=h_{s s}(x, u) \in \mathbb{R}^{p}$ and $x(0)=x_{0} \in \mathbb{R}$, passivity holds if there exists a positive semidefinite function such that:

$$
S(x(\kappa))-S\left(x_{0}\right) \leq \int_{0}^{\kappa} y^{T}(t) u(t) d t
$$

for all input signals $u:[0, \kappa] \rightarrow \mathbb{R}^{p}$, initial states $x_{0} \in \mathbb{R}^{n}$ and $\kappa \in \mathbb{R}_{+}$. Several passive sub-systems can be combined as one single passive system via feedback or parallel interconnections.

In the following we show that the passivity of the controller (13) is not guaranteed. Similar to [12] we solve this problem by inserting a virtual tank to preserve passivity.

Passivity of the proposed unified torque/impedance control (13) w.r.t. $\left[\dot{q},-\tau_{\mathrm{d}}^{\prime}\right]$ can be examined by considering the powerports $\tau_{\mathrm{d}}^{\prime}$ and $\dot{q}$ of the system as

$$
\begin{aligned}
-\dot{q} \tau_{\mathrm{d}}^{\prime} & =-\dot{q}\left(-k_{\mathrm{imp}} \tilde{q}-d_{\mathrm{imp}} \dot{q}+\hat{g}(q)+\tau_{\mathrm{d}, \mathrm{trq}}\right) \\
& =\dot{S}_{I}+d_{\mathrm{imp}} \dot{q}^{2}-\dot{q} \tau_{\mathrm{d}, \mathrm{trq}} \\
& \geq \dot{S}_{I}-\dot{q} \tau_{\mathrm{d}, \mathrm{trq}}
\end{aligned}
$$

where

$$
\tilde{q}=q-q_{\mathrm{d}}
$$

is the displacement from the impedance set-point and

$$
S_{\mathrm{I}}=\frac{1}{2} k_{\mathrm{imp}} \tilde{q}^{2}-V_{\hat{g}}(q)
$$

is the storage function of the impedance controller. The relation between $V_{\hat{g}}(q)$ and $\hat{g}(q)$ is

$$
\hat{g}(q)=\frac{\partial V_{\hat{g}}(q)}{\partial q}
$$

From (15) it can be seen that passivity is potentially violated since $\dot{q} \tau_{\mathrm{d} \text {,trq }}$ is not necessarily negative.

In order to solve this problem, a tank with the state equation

$$
\dot{x}_{\mathrm{t}}=\frac{\beta}{x_{\mathrm{t}}}\left(d_{\mathrm{imp}} \dot{q}^{2}-\gamma \dot{q} \tau_{\mathrm{d}, \mathrm{trq}}\right)+u_{\mathrm{t}}
$$

and an input $u_{\mathrm{t}}$ is introduced. Additionally, the binary keys $\gamma$ and $\beta$ are defined as

$$
\gamma= \begin{cases}1, & \text { if } \dot{q} \tau_{\mathrm{d}, \operatorname{trq}}<0 \rightarrow \text { passivity not violated } \\ 0, & \text { if } \dot{q} \tau_{\mathrm{d}, \mathrm{trq}} \geq 0 \rightarrow \text { passivity violated }\end{cases}
$$

and

$$
\beta= \begin{cases}1, & T_{\mathrm{t}}<T_{\mathrm{u}} \\ 0, & \text { else }\end{cases}
$$

$T_{\mathrm{u}}$ defines an upper tank boundary referring to the tank energy

$$
T_{\mathrm{t}}=\frac{1}{2} x_{\mathrm{t}}^{2}
$$

with the tank state $x_{\mathrm{t}}$. The connection between tank and unified controller will be established by a Dirac structure

$$
\left(\begin{array}{l}
u_{\mathrm{c}} \\
u_{\mathrm{t}}
\end{array}\right)=\left[\begin{array}{cc}
0 & \omega \\
-\omega & 0
\end{array}\right]\left(\begin{array}{l}
y_{\mathrm{c}} \\
y_{\mathrm{t}}
\end{array}\right)
$$

with the output port of the tank $y_{\mathrm{t}}=x_{\mathrm{t}}$, the input $u_{\mathrm{c}}$ and the output port of the controller $y_{\mathrm{c}}=\dot{q}$. The connection $\omega$ between controller and tank is defined by

$$
\omega=\frac{\alpha}{x_{\mathrm{t}}}(1-\gamma) \tau_{\mathrm{d}, \mathrm{trq}} .
$$

Another binary key $\alpha$ is defined as

$$
\alpha= \begin{cases}1, & T_{\mathrm{t}}>T_{1} \\ 0, & \text { else },\end{cases}
$$

with $T_{1}$ being the lower boundary of the tank energy. The tank augmentation will change the control law (13) to

$$
\begin{aligned}
\Gamma & =\gamma \tau_{\mathrm{d}, \operatorname{trq}}+\omega x_{\mathrm{t}} \\
\tau_{\mathrm{d}} & =-k_{\mathrm{imp}} \tilde{q}-d_{\mathrm{imp}} \dot{q}+\hat{g}(q)+\Gamma .
\end{aligned}
$$

Now, the passivity of the unified impedance controller including tank is examined. The new overall storage function 
will now be $S_{\mathrm{u}}=S_{\mathrm{I}}+T_{\mathrm{t}}$. Hence, considering (19) for the case of $T_{\mathrm{t}}<T_{\mathrm{u}}$ we obtain

$$
\begin{aligned}
\dot{S}_{\mathrm{u}} & =\dot{q} k_{\mathrm{imp}} \tilde{q}-\dot{V}_{\hat{g}}(q)+\dot{x}_{\mathrm{t}} x_{\mathrm{t}} \\
& =\dot{q} k_{\mathrm{imp}} \tilde{q}-\dot{V}_{\hat{g}}(q)+\left(\frac{1}{x_{\mathrm{t}}}\left(d_{\mathrm{imp}} \dot{q}^{2}-\gamma \dot{q} \tau_{\mathrm{d}, \mathrm{trq}}\right)-\omega y_{\mathrm{c}}\right) x_{\mathrm{t}} \\
& =\dot{q} k_{\mathrm{imp}} \tilde{q}-\dot{V}_{\hat{g}}(q)+d_{\mathrm{imp}} \dot{q}^{2}-\gamma \dot{q} \tau_{\mathrm{d}, \mathrm{trq}}-\omega \dot{q} x_{\mathrm{t}} \\
& =\dot{q}\left[k_{\mathrm{imp}} \tilde{q}-\hat{g}(q)+d_{\mathrm{imp}} \dot{q}-\gamma \tau_{\mathrm{d}, \mathrm{trq}}-\alpha(1-\gamma) \tau_{\mathrm{d}, \mathrm{trq}}\right] \\
& \leq-\dot{q} \tau_{\mathrm{d}},
\end{aligned}
$$

which not only guarantees the passivity of the unified impedance torque controller w.r.t. $\left[\dot{q},-\tau_{\mathrm{d}}\right]$, but also makes it a lossless system.

It should be mentioned that for $\beta=0$ the passivity condition will still hold, although the system would not be lossless anymore. In case of $\alpha=0$ there would be two situations. If $\gamma=1$, the system would be passive and lossless, however, if $\gamma=0$, the torque controller would be deactivated to guarantee the passivity. Hence, in order to avoid this situation and keeping $\alpha$ to be always 1, the tank should have enough initial energy [12].

In [20] a force controlled pneumatic actuator with a linear feedback is shown to be passive. For a sliding mode controlled pneumatic actuator, as used in this paper, the passivity analysis and possibly necessary modifications are left for future work. Assuming a passive force controlled pneumatic actuator the overall system is passive (see Fig. 3).

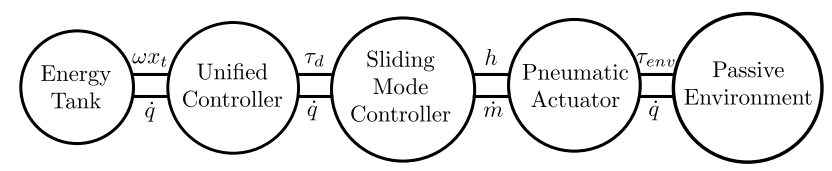

Fig. 3: Abstract port based model of the antagonistic pneumatically actuated system

\section{F. Controller shaping function}

For handling unexpected contact losses, a shaping function $\rho$ is introduced, which provides a smooth transition between torque and impedance control [12]. The defintion of $\Gamma$ is changed to

$$
\Gamma^{\prime}=\Gamma \rho,
$$

where $\rho$ is defined as

$$
\rho= \begin{cases}1 & \tau_{\mathrm{d}} q^{*} \geq 0 \\ \frac{1}{2}\left(1+\cos \left(\pi \frac{q^{*}}{d_{\max }}\right)\right) & \tau_{\mathrm{d}} q^{*}<0 \wedge \\ & \left|q^{*}\right|>0 \wedge\left|q^{*}\right|<d_{\max } \\ 0 & \text { else, }\end{cases}
$$

where $q^{*}=\tilde{q}-\Delta q_{\mathrm{s}}$ and $\Delta q_{\mathrm{s}}=\operatorname{sign}\left(\tau_{\mathrm{d}}\right) \Delta q_{\mathrm{s} 1}$. The parameter $d_{\max }$ adjusts the width of the transition range. The parameter $\Delta q_{\mathrm{s} 1}$ denotes a robustness region, see Fig. 4. Figure 5 shows the full scheme of the controller.

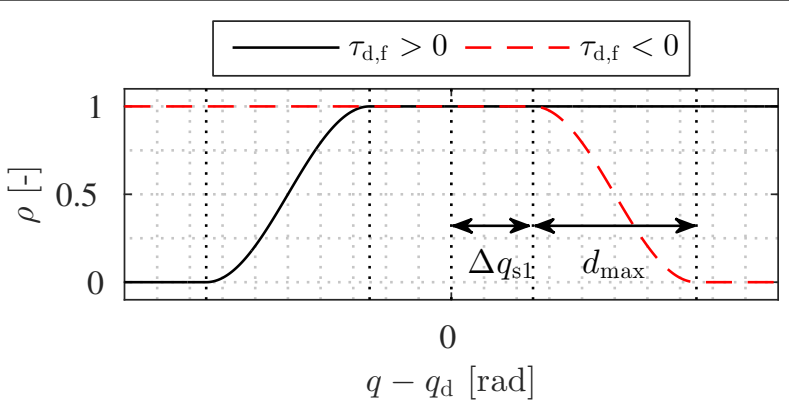

Fig. 4: Shaping function for contact-loss handling

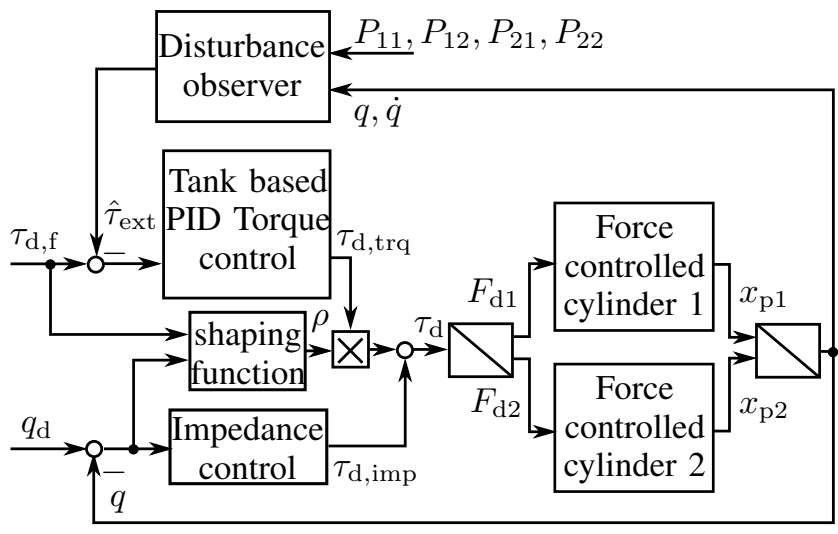

Fig. 5: Torque/impedance control for pneumatic joint

\section{Simulation And EXPerimental Results}

This section presents results for the unified impedance controller applied to a pneumatic joint, comparing simulative and experimental results for various setups. First, the actuator torque tracking is investigated. Second, the contact/noncontact behavior is evaluated.

\section{A. Parameter settings}

The simulation of the controlled pneumatic antagonistic actuator was done based on the model described in Section II and III using MATLAB ${ }^{\circledR} /$ SIMULINK $^{\circledR}$ with a Runge-Kuttasolver and a step size of $10^{-5} \mathrm{~s}$. The contact was modeled as a spring-damper system with stiffness $k_{\mathrm{c}}=50 \mathrm{Nm} / \mathrm{rad}$ and damping $d_{\mathrm{c}}=50 \mathrm{Nms} / \mathrm{rad}$, respectively. The contact becomes active when $q<-0.57^{\circ}$. The impedance damping and stiffness are $d_{\mathrm{imp}}=0.7$ and $k_{\mathrm{imp}}=0.25 \mathrm{Nm} / \mathrm{rad}$ in simulation. For the real experiment the stiffness was chosen to be $k_{\mathrm{imp}}=0.5 \mathrm{Nm} / \mathrm{rad}$. The PID torque control parameters were chosen to be $k_{\mathrm{p}}=0.56, k_{\mathrm{d}}=0.03 \mathrm{~s}, k_{\mathrm{i}}=180 \mathrm{~s}^{-1}$. The momentum observer gain was set to $K_{\mathrm{obs}}=40 \mathrm{~s}^{-1}$. All control operations run at a cycle time of $4 \cdot 10^{-4} \mathrm{~s}$.

The parameters of the shaping function (25) were set to $\Delta q_{s 1}=0.01 \mathrm{rad}$ and $d_{\max }=0.05 \mathrm{rad}$. Parameters used in the state machine for executing the experimental protocol (see IV-D.1) were $\epsilon=\frac{1}{180} \pi, \lambda=2000, \hat{\tau}_{\mathrm{t}}=0.15 \mathrm{Nm}$ and $\Delta t=0.2 \mathrm{~s}$. Further geometrical, mechanical, control and filter parameters can be found in [13].

\section{B. Experimental setup}

Figure 6 depicts the experimental setup for testing the contact/non-contact behavior of the control algorithm. Contact between joint link and environment can be established by 
a contact vehicle which can be shifted linearly on a rail. The experiment was performed with MATLAB $^{\circledR} /$ SIMULINK $^{\circledR}$ on an Intel i7 PC using a real-time Linux kernel. A DAQ system NI 9144 by National Instruments was used for reading/writing analogue data. More details on the used hardware can be found in [13].

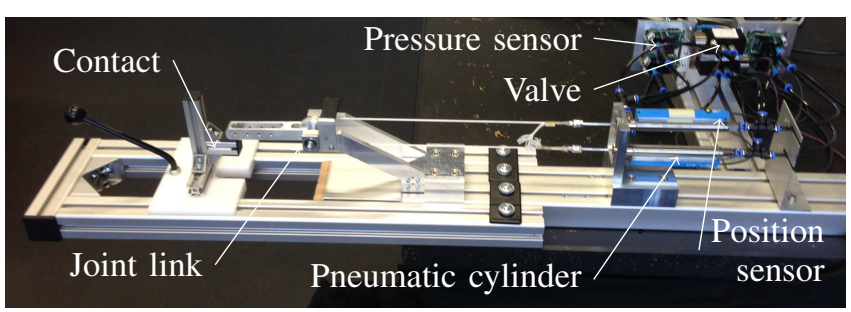

Fig. 6: Experimental setup of the antagonistic pneumatic actuator with a contact slider

\section{Torque and force tracking}

Figure 7 and Fig. 9 show the torque tracking performance in simulation and experiment. After establishing contact tracking was done by (23). A theoretical maximum torque of $\tau_{\max }=0.52 \mathrm{Nm}$ (see (2) and (5)) can be generated by the joint, considering a maximum actuator force of $F_{\mathrm{t} i, \max }=34$ $\mathrm{N}$ (based on 6 bar) and minimum actuator force of $F_{\mathrm{t} i \text {,min }}=$ $5 \mathrm{~N}$ of the opposite actuator to prevent slacking.

The upper row of Fig. 7 shows the torque tracking results for sinusoidal signals of $2 \mathrm{~Hz}$ with different offsets. The offset of the desired torque was increased until a loss in performance could be observed. Simulations and experiments show phase-less tracking for all offsets.

The lower row shows a step function tracking for different offsets. Both simulation and experimental results show an overshoot of $\approx 0.05 \mathrm{Nm}$. Presumably, the experimental noise originates from noise of the pressure sensors in combination with a high momentum observer gain. The latter was chosen as a compromise between filtering and dynamics. In simulation and experiment $\approx 23 \%$ below $\tau_{\max }$ a decreasing control performance can be observed. Probably, the pressure potential between cylinder chamber and tank is too low to provide a sufficient mass flow.

Force control results on actuator level of dataset $(*)$ in Fig. 7 are shown in Fig. 8. Simulation results reveal an almost ideal agreement of desired and actual force. Both tendon forces are in well agreement with a trigonometric function as demanded on joint torque level. Desired forces of the experiment, however, do not match well. When looking at (7) and (13) it becomes clear that due to the superposition of impedance and torque control, sensor influences and observer dynamics disturb the desired torque signal. Furthermore, a constant offset can be observed when comparing tendon forces $F_{\mathrm{t} i}$ and desired force $F_{\mathrm{d} i}$. This might be affected by pressure measurement errors and static friction. Due to the antagonistic arrangement this effect appears to be of little importance, see Fig. 7.

Figure 9 shows the tracking of the desired torque at 5,7 and $8 \mathrm{~Hz}$ in simulation (upper row) and experiment (lower row). Up to $5 \mathrm{~Hz}$ the torque is tracked almost phase-less with a slight overshoot in amplitude of $0.125 \mathrm{Nm}$. From

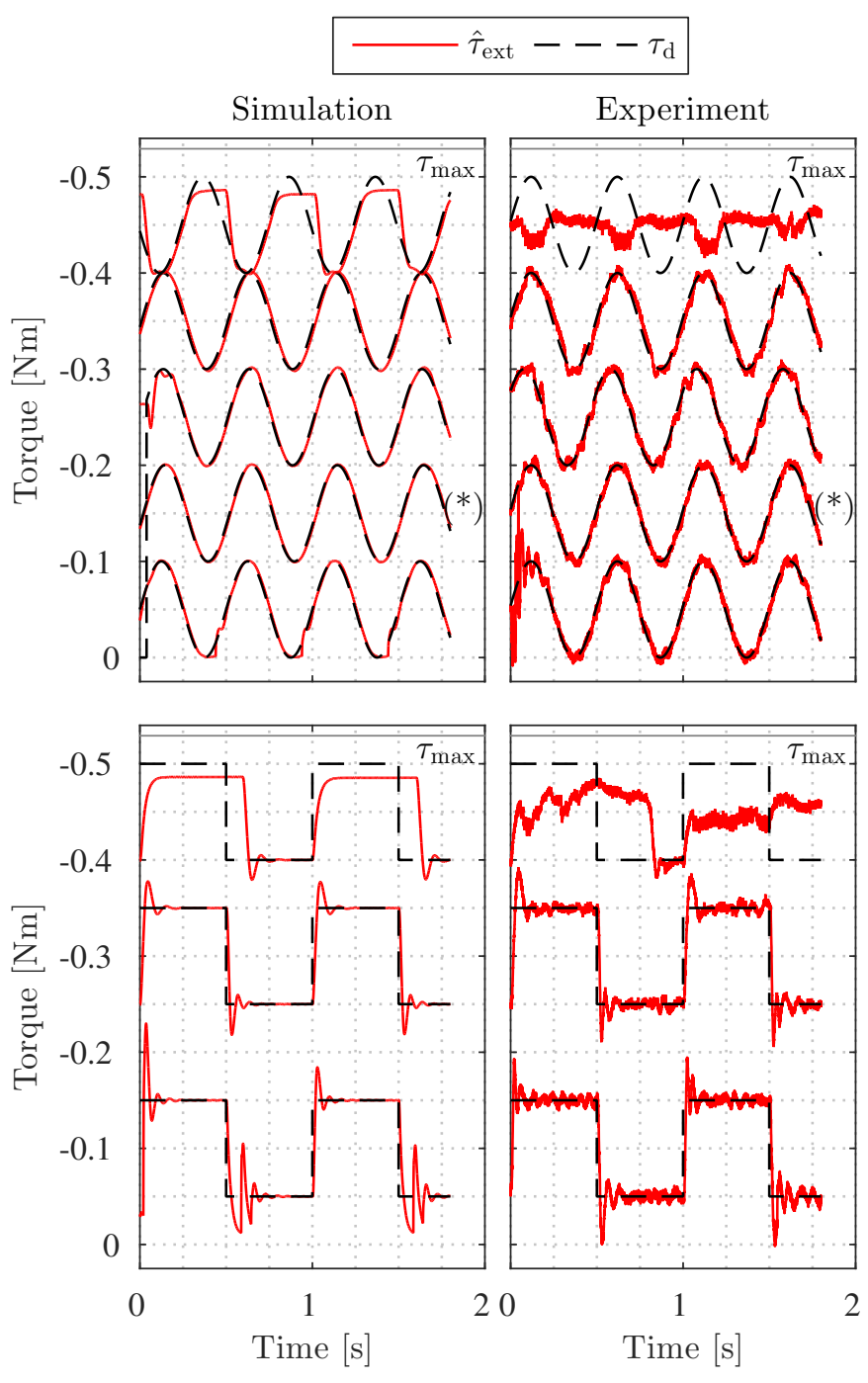

Fig. 7: Experimental and simulation results for torque tracking of sinusoidal and step functions with different contact offsets

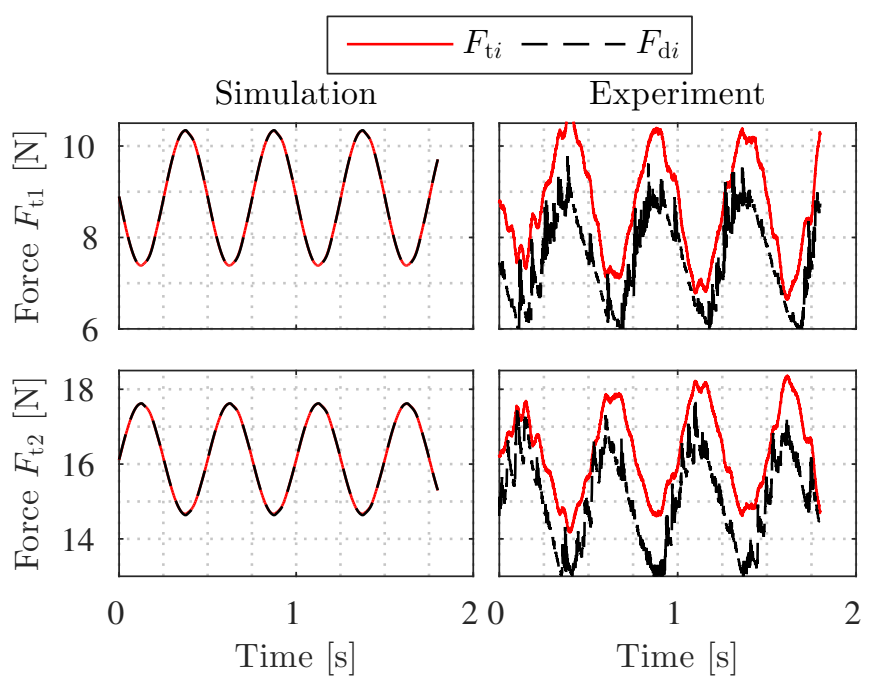

Fig. 8: Experimental and simulation results of cylinder force controllers in dataset $(*)$ from Fig. 7 
$7 \mathrm{~Hz}$ on the control performance decreases in terms of higher amplitude and phase-shift.

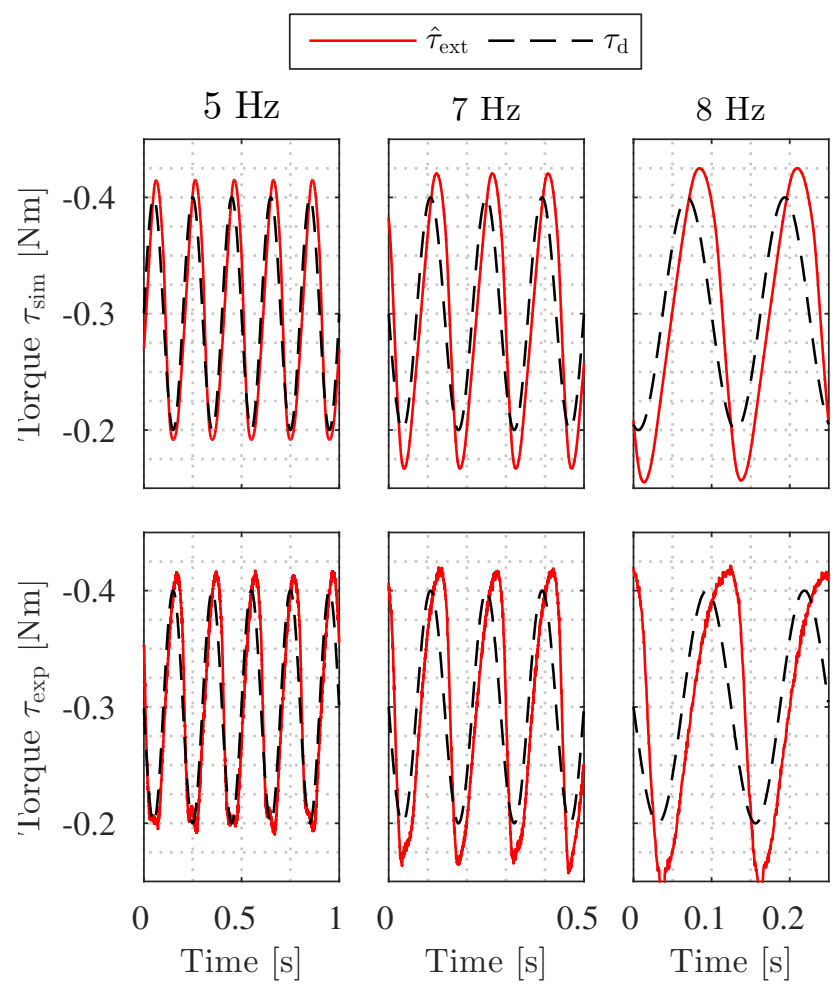

Fig. 9: Experimental and simulation results for sinusoidal torque tracking in contact for different frequencies

\section{Unified torque/impedance controller with contact loss}

1) Experimental protocol: To evaluate the contact/noncontact behavior, the unified impedance controller is evaluated in a more complex experimental protocol that is represented by the state machine in Fig. 10. The simulation and experimental results that are divided into the intervals (a)-(e) are found in Fig. 11. For the analysis the desired trajectory $q_{\mathrm{d} 2}$ is introduced, which is connected in series with $q_{\mathrm{d}} . q_{\mathrm{d} 2}$ performs a repetitive motion (see Fig. 11, blue line). For tracking it yields $q_{\mathrm{d}}=q_{\mathrm{d} 2}$ and depending on the current state of the state machine $q_{\mathrm{d} 2}$ is decoupled from $q_{\mathrm{d}}$.

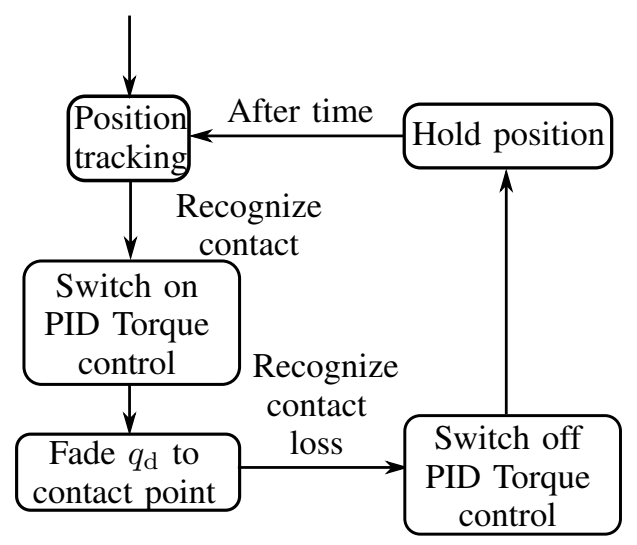

Fig. 10: State machine of the unified impedance controller
The states and transitions of the state machine are described as follows.

- Position tracking (a), (b), (c): the controller fulfills tracking $q_{\mathrm{d}}=q_{\mathrm{d} 2}$ with pure impedance control at predefined stiffness $k_{\text {imp }}$ and damping $d_{\text {imp }}$.

- Recognize contact (c) $\rightarrow$ (d) : the impedance controller is used to establish contact. It is assumed that the impedance controller applies a certain force according to stiffness $k_{\mathrm{imp}}$ and position error $e_{\mathrm{imp}}=q_{\mathrm{d}}-q$ if an obstacle blocks the trajectory of the system. A contact is recognized at time $t_{0}$ if the external torque $\hat{\tau}_{\text {ext }}$ has been exceeded a predefined threshold $\hat{\tau}_{\mathrm{t}}$ for a certain time period $\Delta t$. It yields $q_{\mathrm{d}}=q_{\mathrm{d} 2}$.

- Activate PID torque control (d): the parameter $\alpha$ of (10) is set to 1 to activate PID torque control. Furthermore, the integral part of the controller is reset by setting $t_{1}=t_{0}$. The next state is activated without condition.

- Fade $q_{\mathrm{d}}$ to contact point (d): To avoid fast and unwanted initial motions in case of contact loss, which are caused by the impedance controller for large deflection from the set point, the set point of the impedance control is set close to the contact point $q_{\mathrm{c}}$ as $q_{\mathrm{d}}=q_{\mathrm{c}}-\epsilon$. $q_{\mathrm{c}}$ is the measured position at $t_{0} . \epsilon$ is a small angle to ensure contact until the PID torque controller has reached the desired torque $\tau_{\mathrm{d}, \mathrm{f}}$. A possible fading function

$$
\begin{aligned}
q_{\mathrm{d}} & =\left(q_{\mathrm{c}}-\epsilon-q_{\mathrm{d}, t 0}\right) f(t)+q_{\mathrm{d}, t 0} \\
f(t) & =\left(1-e^{-\lambda\left(t-t_{0}\right)}\right)^{3}
\end{aligned}
$$

is applied to obtain a continuous transition to the new set point. $q_{\mathrm{d}, t 0}$ is the desired angle at time of contact recognition. $\lambda$ affects the widths of the fading zone. $q_{\mathrm{d}}$ remains at this position until the end of (e).

- Recognize contact loss (d) $\rightarrow$ (e): in case of sudden contact loss the link accelerates in the same direction as the force applied to the surface of the obstacle. As a consequence $q$ passes the intervals $\Delta q_{\mathrm{s} 1}$ and $d_{\max }$ of the shaping function $\rho$, see Fig. 4. The further $q$ travels along $d_{\max }$ the lower the value of $\rho$ becomes. Since $\rho$ is multiplied with the torque controller (22) in (24), the contribution of the torque control is continuously decreased. If $\rho$ has reached zero, the contact loss is recognized and the system transits to the next state.

- Deactivate PID torque control (d) $\rightarrow$ (e): Since it was ensured in the previous state that the torque control had been faded out continuously, switching off the torque control by setting $\alpha=0$ does not affect the system behavior anymore. This will be analyzed in Fig. 12.

- Hold position (e): the system holds the position to show the contact loss handling.

- After time (e) $\rightarrow$ (a): a smooth transition is used to align $q_{\mathrm{d}}$ and $q_{\mathrm{d} 2}$ again.

2) Results: The overall behavior of the unified impedance controller during the aforementioned protocol is invested in simulation, see Fig. 11a. The black line $q_{\mathrm{c}}$ indicates the contact angle and its time of activation. The actions in interval (a) and (b) are in agreement with the previous descriptions. In (c) the endpoint of the desired trajectory $q_{\mathrm{d}}$ is set into the object. Therefore, the impedance controller applies a torque of $0.2 \mathrm{Nm}$ in (d) according to a stiffness of $0.25 \mathrm{Nm} / \mathrm{rad}$ 
This is the author's version of an article that has been published in the ICRA 2017 proceedings. Changes were made to this version by the publisher prior to publication.

The final version of record is available at http://dx.doi.org/10.1109/ICRA.2017.7989149
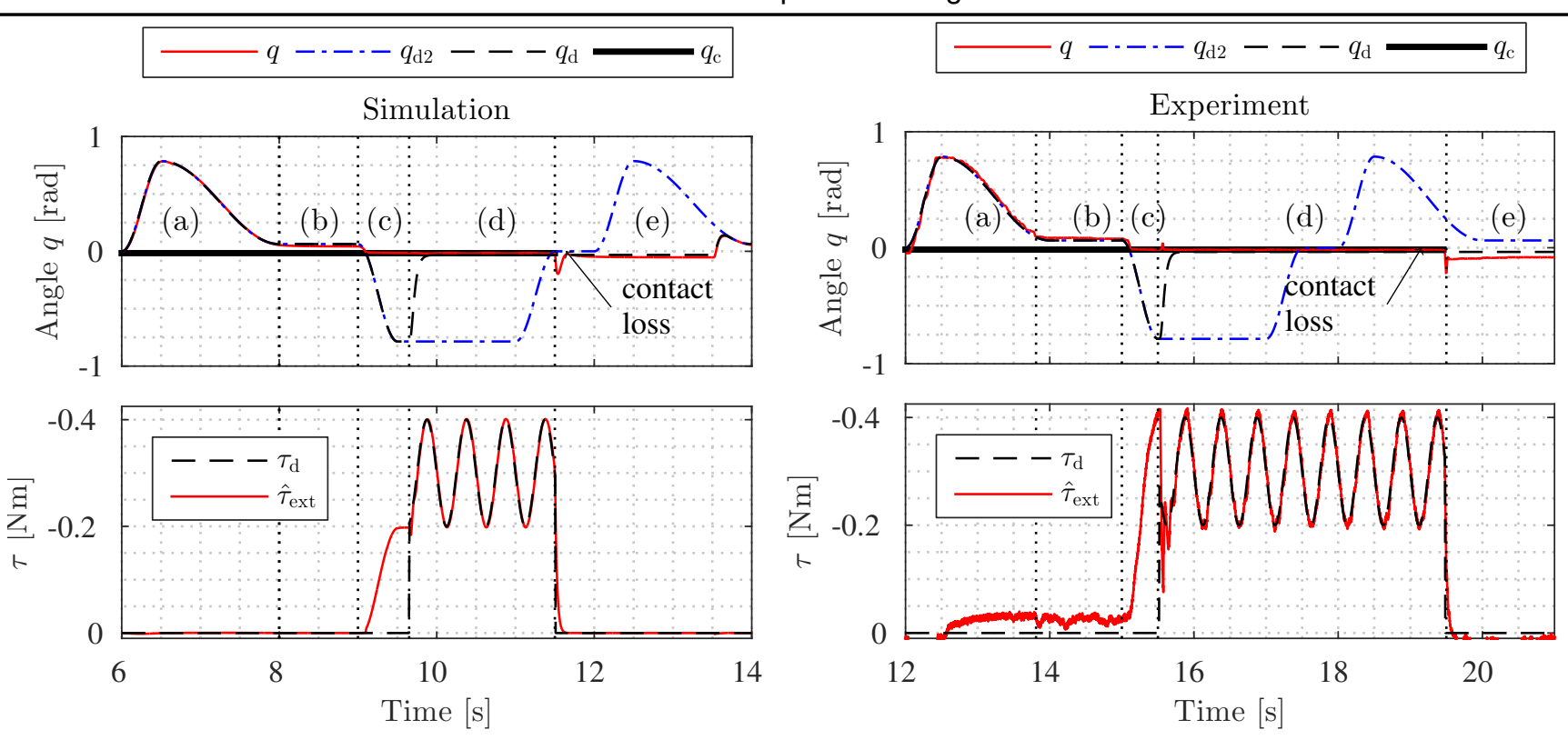

(a)

(b)

Fig. 11: Position tracking with contact/non-contact situation under unified torque/impedance controller

and a position error of $\approx 0.8 \mathrm{rad}$. Since the torque of $0.15 \mathrm{Nm}$ is exceeded ${ }^{3}$ for $\Delta t=0.2 \mathrm{~s}$, the torque controller (10) is activated in addition to the impedance controller (9). Simultaneously, the set-point $q_{\mathrm{d}}$ is faded continuously close to the recognized surface point $q_{\mathrm{c}}-\epsilon$ in order to maintain position in case of contact loss. The torque controller follows a desired torque of $\tau_{\mathrm{d}}=0.1 \sin (2 \pi 2 t)+0.3 \mathrm{Nm}$. The change of the set-point by the impedance controller, which affects a change in applied torque, is handled by the torque controller without noticeable performance loss. Presumably, the integral part of the torque controller can handle changes in the torque fast enough. At $11.5 \mathrm{~s}$ a contact loss occurs, which leads to a fast position change of $-0.17 \mathrm{rad}$ or $-9.7^{\circ}$, respectively. $130 \mathrm{~ms}$ after contact loss the angle $q_{\mathrm{c}}-\epsilon$ is regained and the position is held in (e).

The same experiment is repeated on the experimental setup, see Fig. 6. The contact is established by blocking the trajectory of the joint link with a contact vehicle on a rail. Contact loss is modeled by manually pulling the contact vehicle away from the setup until contact loss occurs. Figure $11 \mathrm{~b}$ depicts the same experiment according to $11 \mathrm{a}$. It provides very similar results in comparison to the simulation. Differences can be observed in the observer torque $\hat{\tau}_{\text {ext }}$, which shows larger errors during tracking operation (a) and (b). This is probably affected by errors in the inertia model or friction since the error is smaller when no motion occurs. Furthermore, the noise of the observer torque is larger than in simulation due to pressure sensor noise. The tracking performance of the torque controller is in well agreement with the results of Fig. 7. When switching to torque control, oscillations in $\hat{\tau}_{\text {ext }}$ and a small overshoot in $q$ can be observed at $15.5 \mathrm{~s}$. This can be explained by a short

\footnotetext{
${ }^{3}$ Duration of $\Delta t$ as well as depth of $q_{\mathrm{c}}-q_{\mathrm{d}}$ are chosen in a way to illustrate the concept. In an application they would certainly be selected much smaller.
}

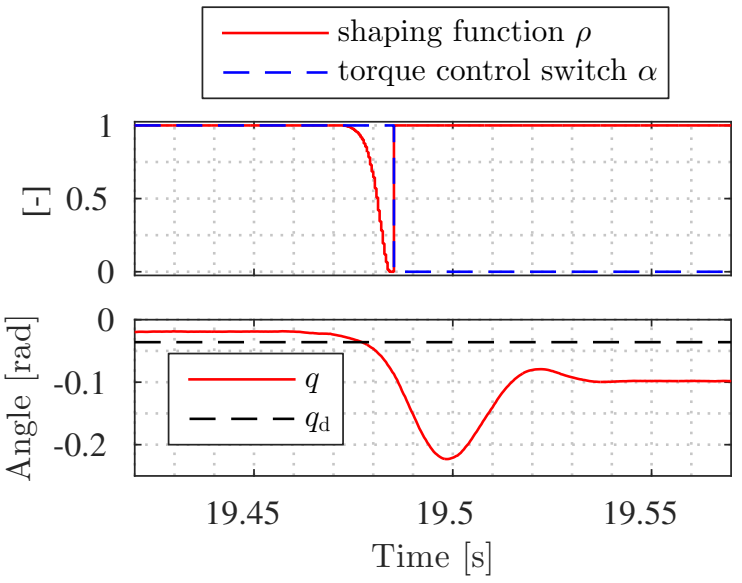

Fig. 12: Experimental result of an enlarged section of Fig. $11 \mathrm{~b}$ showing the behavior of the shaping function in a contact loss situation

misbalance between actuator forces due to a discontinuous input signal affected by the time behavior of momentum observer PID torque control and pneumatic actuators.

Figure 12 investigates the contact loss between $19.45 \mathrm{~s}$ and $19.55 \mathrm{~s}$ of Fig. $11 \mathrm{~b}$ in more detail. The maximum angular deflection amounts to $-0.062 \mathrm{rad}$ or $-3.56^{\circ}$, respectively. $70 \mathrm{~ms}$ after contact loss occurs the system reaches a resting position. The difference to the desired angle $q_{\mathrm{d}}$ may be explained by friction. The upper row of Fig. 11b shows the result of the shaping function $\rho$. It is evident that the shaping function decays smoothly to zero before torque control is switched off. 


\section{CONCLUSION}

The results of this paper show that the concept of unified impedance control concept can be applied to antagonistic pneumatically actuated robot joints. The controller shows good results for both tracking and torque control operations. The reaction to contact loss is handled fast and accurately. In future work the concept shall be applied to multi-joint pneumatic structures on end-effector level so that full use of the shaping function in multiple directions can be made. Furthermore, we will elaborate on the passivity analysis and extend it also to the piston level force controller.

\section{REFERENCES}

[1] G. A. Pratt and M. M. Williamson, "Series elastic actuators," in Intelligent Robots and Systems 95.'Human Robot Interaction and Cooperative Robots', Proceedings. 1995 IEEE/RSJ International Conference on, vol. 1, pp. 399-406, IEEE, 1995.

[2] R. Schiavi, G. Grioli, S. Sen, and A. Bicchi, "Vsa-ii: a novel prototype of variable stiffness actuator for safe and performing robots interacting with humans," in Robotics and Automation, 2008. ICRA 2008. IEEE International Conference on, pp. 2171-2176, IEEE, 2008.

[3] Y. Zhu and E. Barth, "Impedance control of a pneumatic actuator for contact tasks," in Robotics and Automation, 2005. ICRA 2005. Proceedings of the 2005 IEEE International Conference on, pp. 987992, April 2005.

[4] B. Taheri, D. Case, and E. Richer, "Force and stiffness backsteppingsliding mode controller for pneumatic cylinders," IEEE/ASME Transactions on Mechatronics, pp. 1-11, 2013.

[5] B. Ugurlu, P. Forni, C. Doppmann, and M. Jun, "Torque and variable stiffness control for antagonistically driven pneumatic muscle actuators via a stable force feedback controller," in 2015 IEEE/RSJ International Conference on Intelligent Robots and Systems (IROS), 2015.

[6] N. Hogan, "Impedance control: An approach to manipulation," in American Control Conference, 1984, pp. 304-313, IEEE, 1984.

[7] B. Siciliano and O. Khatib, Springer handbook of robotics. Springer Science \& Business Media, 2008.

[8] G. Zeng and A. Hemami, "An overview of robot force control," Robotica, vol. 15, pp. 473-482, Sept. 1997.

[9] X. Shen and M. Goldfarb, "Simultaneous force and stiffness control of a pneumatic actuator," Journal of Dynamic Systems, Measurement, and Control, vol. 129, no. 4, pp. 425-434, 2007.

[10] N. H. Arbab and F. Najafi, "Stability and performance of pneumatic actuators in impedance control," Proceedings of the Institution of Mechanical Engineers, Part I: Journal of Systems and Control Engineering, vol. 228, no. 7, pp. 476-485, 2014.

[11] T. Noritsugu and T. Tanaka, "Application of rubber artificial muscle manipulator as a rehabilitation robot," Mechatronics, IEEE/ASME Transactions on, vol. 2, pp. 259-267, Dec 1997.

[12] C. Schindlbeck and S. Haddadin, "Unified passivity-based cartesian force/impedance control for rigid and flexible joint robots via taskenergy tanks," in IEEE International Conference on Robotics and Automation (ICRA2015), (Seattle, USA), 2015.

[13] A. Toedtheide, T. Lilge, and S. Haddadin, "Antagonistic impedance control for pneumatically actuated robot joints," IEEE Robotics and Automation Letters, vol. 1, pp. 161-168, Jan 2016.

[14] E. Richer and Y. Hurmuzlu, "A high performance pneumatic force actuator system: Part i nonlinear mathematical model," Journal of dynamic systems, measurement, and control, vol. 122, no. 3, pp. 416$425,2000$.

[15] A. De Luca and R. Mattone, "Actuator failure detection and isolation using generalized momenta," in Robotics and Automation, 2003. Proceedings. ICRA'03. IEEE International Conference on, vol. 1 , pp. 634-639, IEEE, 2003.

[16] A. De Luca and R. Mattone, "Sensorless robot collision detection and hybrid force/motion control," in Proceedings of the 2005 IEEE international conference on robotics and automation, pp. 999-1004, IEEE, 2005.

[17] E. Richer and Y. Hurmuzlu, "A high performance pneumatic force actuator system: part ii nonlinear controller design," Journal of dynamic systems, measurement, and control, vol. 122, no. 3, pp. 426-434, 2000.

[18] R. M. Murray, A Mathematical Introduction to Robotic Manipulation. CRC press, 1994.

[19] T. Wimböck, Controllers for Compliant Two-Handed Dexterous Manipulation, vol. 17 of Modellierung und Regelung komplexer dynamischer Systeme. Aachen: Shaker Verlag, 2013.
[20] Y. Zhu and E. J. Barth, "Passivity-based impact and force control of a pneumatic actuator," Journal of Dynamic Systems, Measurement, and Control, vol. 130, no. 2, p. 024501, 2008. 